\title{
Synthesis and Characterization of $\mathrm{WS}_{2}$ Inorganic Nanotubes with Encapsulated/Intercalated CsI
}

\author{
Sung You Hong ${ }^{1}$, Ronit Popovitz-Biro ${ }^{2}$, Gerard Tobias ${ }^{3}$, Belén Ballesteros ${ }^{3}$, Benjamin G. Davis ${ }^{1}$, Malcolm \\ L. H. Green ${ }^{3}$, and Reshef Tenne ${ }^{4}(\varangle)$ \\ ${ }^{1}$ Department of Chemistry, Chemistry Research Laboratory, University of Oxford, Mansfield Road, Oxford, OX1 3TA, UK \\ ${ }^{2}$ Electron Microscopy Unit, Weizmann Institute of Science, Rehovot 76100, Israel \\ ${ }^{3}$ Inorganic Chemistry Laboratory, University of Oxford, South Parks Road, Oxford, OX1 3QR, UK \\ ${ }^{4}$ Department of Materials and Interfaces, Weizmann Institute of Science, Rehovot 76100, Israel \\ Received: 11 December 2009 / Revised: 1 January 2010 / Accepted: 4 January 2010 \\ (C) The Author(s) 2010. This article is published with open access at Springerlink.com
}

\begin{abstract}
$\mathrm{WS}_{2}$ nanotubes have been filled and intercalated by molten phase caesium iodide. The presence of caesium iodide inside the $\mathrm{WS}_{2}$ nanotubes has been determined using high-resolution transmission electron microscopy (HRTEM) coupled with electron energy-loss spectroscopy (EELS) and energy-dispersive X-ray spectroscopy (EDS). Noticeably, a Moiré pattern was observed due to the interference between encapsulated CsI and $\mathrm{WS}_{2}$ layers. The intercalation of CSI into the host concentric $\mathrm{WS}_{2}$ lattices resulted in an increase in the interplanar spacing.
\end{abstract}

\section{KEYWORDS}

Inorganic nanotubes, transmission electron microscopy, intercalation, encapsulation

\section{Introduction}

The discovery of carbon nanotubes (CNTs) initiated the exploration and discovery of inorganic layered compounds with related tubular structures [1]. A wide variety of metal oxides, metal halides, and other materials have been introduced into CNTs by capillary wetting [2-5]. For example, Green and coworkers reported one-dimensional crystals of various metal halides in both multi-walled and single-walled carbon nanotube capillaries $[3,6,7]$. By analogy, filling by use of nanocapillaries was also explored for the inorganic $\mathrm{WS}_{2}$ nanotubes. In 1992, Tenne and coworkers first showed that $\mathrm{WS}_{2}$ material of nanometer scale in a platelet form could spontaneously form closed cage structures known as inorganic fullerene-like (IF) nanoparticles and inorganic nanotubes [8], which are akin to carbon fullerenes and carbon nanotubes. The structures of graphite and $\mathrm{WS}_{2}$ belong to the space group, $P 6_{3} / m m c$. However, the structure of $\mathrm{WS}_{2}$ is more complex than that of graphite. The $\mathrm{WS}_{2}$ layer contains a layer of metal atoms, sandwiched between two sulphur layers, with the metal atom bonded to six sulphur atoms in a trigonal prismatic coordination [9]. Folding and bonding of edge atoms on the periphery of the quasi two-dimensional planar nanostructure leads to the formation of closed cages with polyhedral or nanotubular shapes [1]. The inner diameters of $\mathrm{WS}_{2}$ inorganic nanotubes range between $15-30 \mathrm{~nm}$ and the tips are inherently open-ended [10], so this nanotubular structure is suitable for capillary filling using molten metal halides.

Address correspondence to reshef.tenne@weizmann.ac.il 


\section{Experimental}

\subsection{Synthesis of CsI@WS ${ }_{2}$ NTs}

$\mathrm{WS}_{2}$ nanotubes and fullerene-like nanoparticles were prepared in a fluidized-bed reactor [10]. A mixture of the multi-walled $\mathrm{WS}_{2}$ nanotubes $(5 \%)$ in IF-WS nanoparticle powder (30 mg) and CsI (120 mg, Acros, $99.9 \%$, m.p. $621^{\circ} \mathrm{C}$ ) were ground using a mortar and pestle and transferred to a silica quartz ampoule $10 \mathrm{~mm}$ in diameter. The mixture was dried under high vacuum (approx. $10^{-4}-10^{-5}$ torr) for $2 \mathrm{~h}$, and the ampoule was then sealed. The ampoule was heated to $640{ }^{\circ} \mathrm{C}$ for $4 \mathrm{~h}$. The furnace was cooled to room temperature overnight.

\subsection{TEM analysis of CsI@WS ${ }_{2}$ NTs}

The product was sonicated in ethanol, placed on a carbon/collodion-coated $\mathrm{Cu}$ grid, and analyzed by transmission electron microscopy (TEM) (Philips CM-120, $120 \mathrm{kV}$ ) and high-resolution TEM (HRTEM) (FEI Tecnai F-30 with electron energy-loss spectroscopy (EELS) or JEOL JEM-3000F field emission gun, $300 \mathrm{kV}$ ). Images were acquired digitally on a Gatan model 794 $(1000 \times 1000)$ charge coupled device (CCD) camera, the magnification of which was calibrated with the Si [110] lattice spacing. Energy-dispersive X-ray spectroscopy (EDS) was performed with an electron probe $0.5 \mathrm{~nm}$ in diameter.

\section{Results and discussion}

Compared to the carbon nanotubes, the inorganic $\mathrm{WS}_{2}$ nanotubes have heavier elements $(\mathrm{W}, \mathrm{Z}=74$; $S, Z=16)$. Therefore, there is a severe limitation to the visualization of light elements, which may be filled within $\mathrm{WS}_{2}$ nanotubes. For this reason caesium iodide was the metal halide of choice, because of the strong electron-scattering power of Cs $(Z=55)$ and $I(Z=53)$ which it was anticipated would allow observation of CsI crystal structures within the $\mathrm{WS}_{2}$ nanotubes using HRTEM analysis. We note that CSI is one of a group of alkali halides that adopt the $\mathrm{Pm} 3 m \mathrm{CsCl}$ structural archetype ( $a=0.4520 \mathrm{~nm}$ ) [11]. An HRTEM micrograph of the resulting composite is presented in Fig. 1, where the (110) lattice plane of CsI with lattice interplanar spacing of $0.32 \mathrm{~nm}$ is observed within the core of the
$\mathrm{WS}_{2}$ nanotubes, as confirmed by the fast Fourier transform (FFT) pattern. Remarkably, a Moiré pattern was found along the $\mathrm{WS}_{2}$ nanotube surface. The Moiré $d$ spacing was calculated based on the two lattice spacings with a mismatch of 0.049 or $0.056 \mathrm{~nm}$ from the (100) or (101) spacings of the $\mathrm{WS}_{2}$ crystal, respectively. The calculated Moiré spacing was $1.5-1.8 \mathrm{~nm}$, which was in good agreement with the corresponding Moiré spacing measured from the line profile recorded on the HRTEM image (ca. $1.6 \mathrm{~nm}$ ). Stacking of the caesium iodide crystals inside the nanocapillary exhibited various orientations, such as (110) and (100) lattice planes (see Figs. S-1 and S-2 in the Electronic Supplementary Material (ESM)). Further, EELS analysis performed (Fig. S-3 in the ESM) on such nanotubes gave a Cs:I ratio fairly close to $1: 1$, consistent with the existence of CSI layers. EDS analysis also confirmed

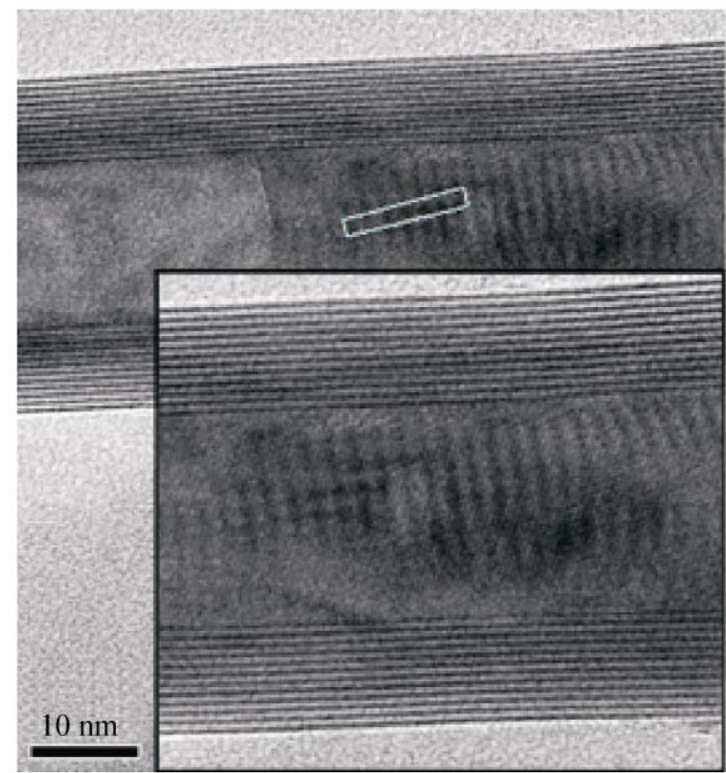

(a)

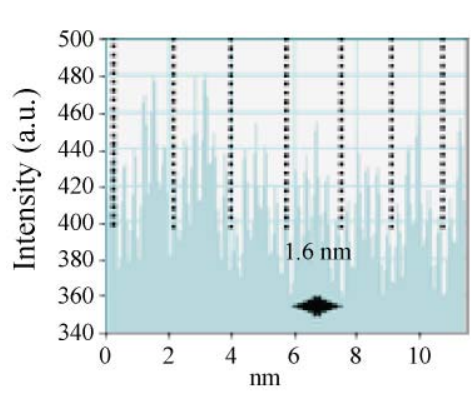

(b)

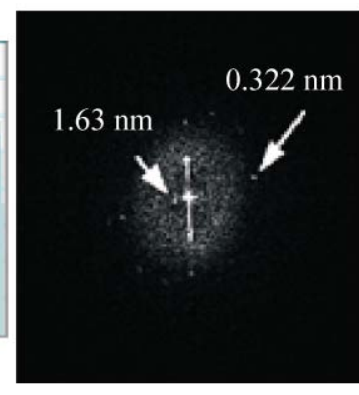

(c)
Figure 1 (a) Moiré pattern of HRTEM image of CsI filled $\mathrm{WS}_{2}$ nanotube; (b) line profile integrated along the region enclosed in the rectangle; (c) FFT pattern 
the presence of W, S, Cs, and I (Fig. S-3 in the ESM). In addition to the nanotubes filled with CsI rod-like crystals, occasionally, some of $\mathrm{WS}_{2}$ nanotubes $(<10 \%)$ showed lattice expansion of the concentric $\mathrm{WS}_{2}$ layers and revealed a substantial increase (ca. $0.7 \mathrm{~nm}$ ) in the interlayer spacing (Fig. 2). While the encapsulation is induced by nanocapillarity, the intercalation mechanism is not clearly understood. Presumably, it involves diffusion from both inner and outer walls of $\mathrm{WS}_{2}$ nanotubes: such a significant increase in layer spacing can be attributed to the uptake of water into these intercalated layers during exposure to the atmosphere. A similar behavior of hydration of intercalated layers has been described earlier for $\mathrm{WS}_{2}$ materials intercalated by alkali metals $[12,13]$. Also, alkali metal atom intercalated CNTs show a similar hydration expansion [14]. The occurrence of intercalation of CsI into the layers may be associated with the weak interlayer van der Waals interaction between the tungsten metal dichalcogenide layers [15]. However, more extensive studies are required to explain the interlayer expansion.

\section{Conclusions}

We have shown that filling of nanotubes by nanocapillarity is not limited to carbon nanotubes but can be applied to the inorganic nanotubes of $\mathrm{WS}_{2}$. Molten phase wetting with CsI resulted in the formation of one-dimensional crystal structures inside $\mathrm{WS}_{2}$ nanocapillaries. A Moiré pattern was observed in the

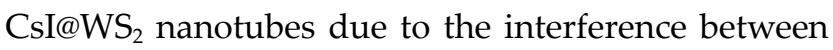
$\mathrm{CsI}$ and $\mathrm{WS}_{2}$ crystal layers. Moreover, the observation of an increase (ca. $0.7 \mathrm{~nm}$ ) of the interlayer spacing between concentric $\mathrm{WS}_{2}$ layers of inorganic nanotubes indicates that intercalation has occurred and the expansion with the CsI is larger than that $(0.3-0.5 \mathrm{~nm})$ found for alkali metal intercalated IF-WS ${ }_{2}$ [15]. Unlike the previously described $\mathrm{PbI}_{2} @ \mathrm{WS}_{2}$ nanotubes $\left(\mathrm{PbI}_{2}\right.$, two-dimensional layered) having a core-shell nanotubular structure [16], the CsI@WS ${ }_{2}$ nanotube (CsI, 3-D $\mathrm{CsCl}$ archetype) has a one-dimensional stacking inside the $\mathrm{WS}_{2}$ nanotube and also intercalation between concentric $\mathrm{WS}_{2}$. This suggests that metal halides do not interact strongly with the $\mathrm{WS}_{2}$ layers but instead

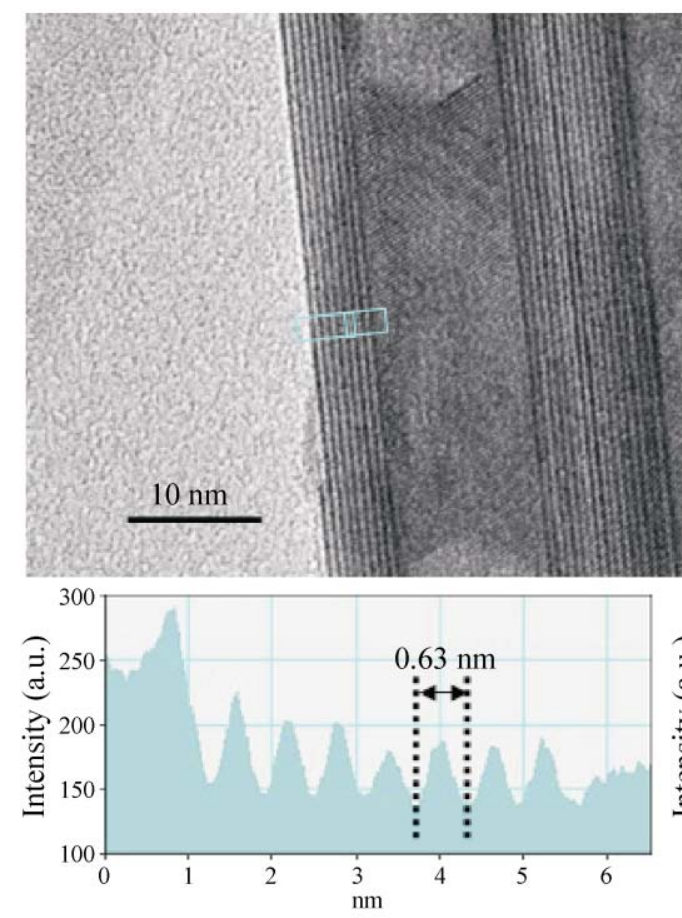

(a)

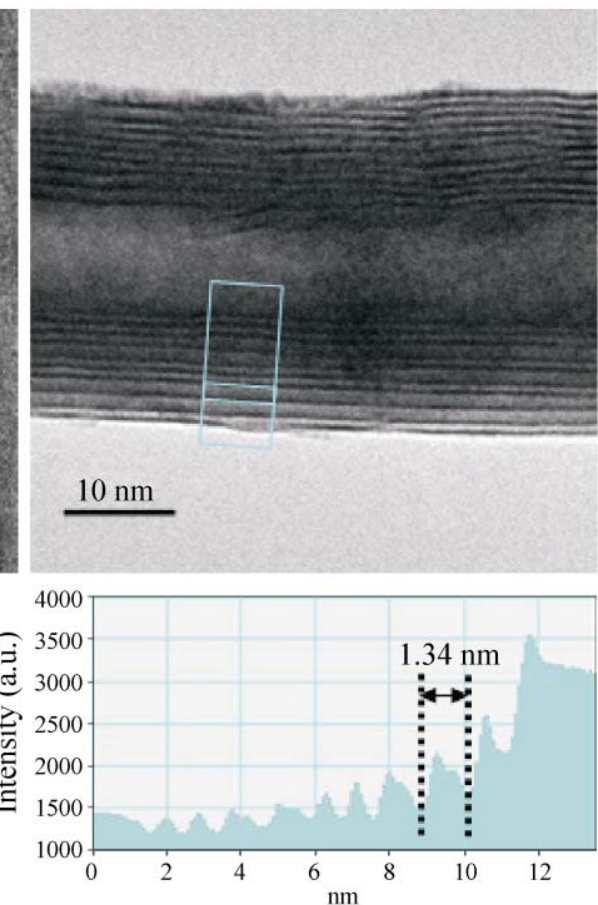

(b)

Figure 2 HRTEM images with line profiles: (a) non-intercalated $\mathrm{WS}_{2}$ nanotube with encapsulated CsI; (b) $\mathrm{WS}_{2}$ nanotube with intercalated CsI 
adopt their preferred ionic structures.

\section{Acknowledgements}

This work was supported by the Israel Science Foundation, European Research Council (ERC) grant (No. INTIF 226639); H. Perlman Foundation and the Cherna Moskowitz Center for Nano and Bio-Nano imaging. We thank Dr. Rita Rosentsveig for the supply of $\mathrm{WS}_{2}$ inorganic nanotubes. G. T. acknowledges support through FP7 European Community Marie Curie European Reintegration Grant (ERG) (No. PERG04-GA-2008-239303) and B. B. a contract from Ministry of Science and Innovation (Ministerio de Ciencia e Innovación, MICINN) Spain. S. Y. H was supported by a Samsung Corporation fellowship (2005-2009). B. G. D. is a Royal Society-Wolfson Research Merit Award recipient and is also supported by an Engineering and Physical Sciences Research Council of the UK (EPSRC) Platform Grant (No. EPSRC EP/E000614/1).

Electronic Supplementary Material: Additional HRTEM images and details of elemental analysis by EDS and EELS are available in the online version of this article at http://dx.doi.org/10.1007/s12274-010-1018-0 and are accessible free of charge.

Open Access: This article is distributed under the terms of the Creative Commons Attribution Noncommercial License which permits any noncommercial use, distribution, and reproduction in any medium, provided the original author(s) and source are credited.

\section{References}

[1] Tenne, R. Inorganic nanotubes and fullerene-like nanoparticles. Nat. Nanotechnol. 2006, 1, 103-111.

[2] Ajayan, P. M.; Iijima, S. Capillarity-induced filling of carbon nanotubes. Nature 1993, 361, 333-361.

[3] Tsang, S. C.; Chen, Y. K.; Harris, P. J. F.; Green. M. L. H. A simple chemical method of opening and filling carbon nanotubes. Nature 1994, 372, 159-162.

[4] Ugarte, D.; Chatelain, A.; de Heer, W. A. Nanocapillarity and chemistry in carbon nanotubes. Science 1996, 274, 1897-1899.
[5] Takenobu, T.; Takano, T.; Shiraishi, M.; Murakami, Y.; Ata, M.; Kataura, H.; Achiba, Y.; Iwasa, Y. Stable and controlled amphoteric doping by encapsulation of organic molecules inside carbon nanotubes. Nat. Mater. 2003, 2, 683-688.

[6] Sloan, J.; Kirkland, A. I.; Hutchison, J. L.; Green, M. L. H. Integral atomic layer architectures of $1 \mathrm{D}$ crystals inserted into single walled carbon nanotubes. Chem. Commun. 2002, 1319-1332.

[7] Meyer, R. R.; Sloan, J.; Dunin-Borkowski, R. E.; Kirkland, A. I.; Novotny, M. C.; Bailey, S. R.; Hutchison, J. L.; Green, M. L. H. Discrete atom imaging of one-dimensional crystals formed within single-walled carbon nanotubes. Science 2000, 289, 1324-1326.

[8] Tenne, R.; Margulis, L.; Genut, M.; Hodes, G. Polyhedral and cylindrical structures of tungsten disulphide. Nature 1992, 360, 444-446.

[9] Kertesy, M.; Hoffman, R. Octahedral vs. trigonal-prismatic coordination and clustering in transition-metal dichalcogenides. J. Am. Chem. Soc. 1984, 106, 3453-3460.

[10] Rosentsveig, R.; Margolin, A.; Feldman, Y.; Popovitz-Biro, R.; Tenne, R. $\mathrm{WS}_{2}$ nanotube bundles and foils. Chem. Mater. 2002, 14, 471-473.

[11] Kelly, A.; Groves, G. W.; Kidd, P. Crystallography and Crystal Defects; Wiley: Chichester, $2^{\text {nd }}$ ed., 2000.

[12] Wypych, F.; Schollhorn, R. 1T $\mathrm{MoS}_{2}$, a new metallic modification of molybdenum disulfide. J. Chem. Soc., Chem. Commun. 1992, 1386-1388.

[13] Schellenberger, A.; Schlaf, R.; Pettenkofer, C.; Jaegerman, W. Synchrotron-induced surface-photovoltage saturation at intercalated $\mathrm{Na} / \mathrm{WSe}_{2}$ interfaces. Phys. Rev. B 1992, 45, 3538-3545.

[14] Zhou, O.; Fleming, R. M.; Murphy, D. W.; Chen, C. H.; Haddon, R. C.; Ramirez, A. P.; Glarum, S. H. Defects in carbon nanostructures. Science 1994, 263, 1744-1747.

[15] Zak, A.; Feldman, Y.; Lyakhoviskaya, V.; Leitus, G.; Popovitz-Biro, R.; Wachtel, E.; Cohen, H.; Reich, S.; Tenne, R. Alkali metal intercalated fullerene-like $\mathrm{MS}_{2}(\mathrm{M}=\mathrm{W}, \mathrm{Mo})$ nanoparticles and their properties. J. Am. Chem. Soc. 2002, 124, 4747-4758.

[16] Kreizman, R.; Hong, S. Y.; Sloan, J.; Popovitz-Biro, R.; Albu-Yaron, A.; Tobias, G.; Ballesteros, B.; Davis, B. G.; Green, M. L. H.; Tenne, R. Core-shell $\mathrm{PbI}_{2} @ \mathrm{WS}_{2}$ inorganic nanotubes from capillary wetting. Angew. Chem. Int. Ed. 2009, 48, 1230-1233. 